\title{
On the Quasi-periodic Gravitational Modulation of Polymerization Front with Solid Product
}

\author{
Karam Allali $^{1, a}$, Saadia Assiyad ${ }^{1, b}$, and Mohamed Belhaq ${ }^{2, c}$ \\ 1 University Hassan II-Mohammedia, FSTM, Department of Mathematics, PO Box Mohammadia-Morocco \\ 2 University Hassan II-Casablanca, FS, Department of Physics, Casablanca-Morocco
}

\begin{abstract}
The quasi-periodic gravitational modulation of polymerization front with solid product is studied in this paper. The model we consider includes the heat equation, the equation for the concentration and the NavierStokes equations under the Boussinesq approximation. Linear stability analysis of the problem is fulfilled and the convective instability boundary is determined using numerical simulation. Results shown that the convective instability threshold depends strongly on the amplitudes and on the frequencies ratio.
\end{abstract}

\section{Introduction}

Frontal polymerization is the process of converting monomer to polymer via a narrow located zone, called reaction front [1]. In this paper, we are interested in the influence of quasi-periodic (QP) gravitational modulation on the convective instability of polymerization front with solid product.

The influence of periodic gravitational modulation on the convective instability of reaction front was studied in [2], while the effect of QP gravitational modulation on convective instability of reaction fronts in porous media was examined in [3]. In contrast to the case of a periodic modulation which was extensively studied, only few works have been devoted to study the effect of a QP vibration on the convective instability. For instance, Boulal et al. [4] reported on the effect of a QP gravitational modulation on the stability of a heated fluid layer. It was shown that the frequencies ratio of QP vibration strongly influences the convective instability threshold. In [5] the influence of QP gravitational modulation on convective instability in HeleShaw cell was also analyzed. Similar study has been made for thermal instability in horizontal Newtonian magnetic liquid layer with non-magnetic rigid boundaries in the presence of a vertical magnetic field and a QP modulation [6]. In these works $[4,6]$, the original problem is systematically reduced to a QP Mathieu equation using Galerkin method truncated to the first order. Since the Floquet theory cannot be applied in the QP forcing case, the approach used to obtain the marginal stability curves was based on the application of the harmonic balance method combined Hill's determinants $[7,8]$.

Recently, the influence of QP gravitational vibration on convective instabilities of reaction fronts in porous media was investigated [3]. It was shown that the convection instability of reaction fronts in porous media can be controlled and the reaction fronts may remain stable in some regions and for certain combinations of the amplitudes and

\footnotetext{
a e-mail: allali@hotmail.com

b e-mail: assiyad.saadia@gmail.com

c e-mail: belhaq@yahoo.fr
}

the frequencies ratio of the QP vibration. This result may have specific application arising in some physical problems, as for instance, frontal polymerization [9] or the environmental pollution [10]. It is worth noticing that $\mathrm{QP}$ vibration may result from a simultaneous existence of a basic vibration applied to the system with a frequency $\sigma_{1}$ and of an additional residual vibration having a frequency $\sigma_{2}$, such that $\sigma_{1}$ and $\sigma_{2}$ are incommensurate.

While in [3] attention was focused on the problem of reaction front propagation in porous media with liquid reactant and liquid product considering Darcy equation, the present paper reports on the problem of polymerization front with liquid reactant and solid product considering Navier-Stokes equations.

In order to study the influence of QP gravitational modulation on convective instability of polymerization front with solid product, we consider that the acceleration $b$ acting on the fluid is given by $g+b(t)$, where $g$ is the gravity acceleration, $b(t)=\lambda_{1} \sin \left(\sigma_{1} t\right)+\lambda_{2} \sin \left(\sigma_{2} t\right)$ and $\lambda_{1}, \lambda_{2}$ and $\sigma_{1}, \sigma_{2}$ are the amplitudes and the frequencies of the QP vibration, respectively.

Because one cannot truncate the problem under consideration to a QP Mathieu equation using Galerkin method as well as the Floquet theory, the marginal stability curves are obtained by using the approximately narrow zone method (Frank-Kamenetskii method) and the matched asymptotic expansions to obtain the interface problem to be solved by numerical simulations.

The paper is organized as follows. We first introduce the model in the next section, then we perform a linear stability analysis and we analyze the interface problem in section 3. Section 4 deals with numerical simulation and section 5 concludes the work.

\section{Reaction front model}

The propagation of reaction polymerization front is modelled by the following system of equations:

$$
\frac{\partial T}{\partial t}+(v \cdot \nabla) T=\kappa \Delta T+q W,
$$




$$
\begin{gathered}
\frac{\partial \alpha}{\partial t}+(v \cdot \nabla) \alpha=W \\
\frac{\partial v}{\partial t}+(v \cdot \nabla) v=-\frac{1}{\rho} \nabla p+v \Delta v+g\left(1+\lambda_{1} \sin \left(\sigma_{1} t\right)\right. \\
\left.+\lambda_{2} \sin \left(\sigma_{2} t\right)\right) \beta\left(T-T_{0}\right) \gamma, \\
\operatorname{div}(v)=0,
\end{gathered}
$$

with the following boundary conditions:

$$
\begin{aligned}
& z \rightarrow+\infty, \quad T=T_{i}, \quad \alpha=0, \quad \text { and } \quad v=0, \\
& z \rightarrow-\infty, \quad T=T_{b}, \quad \alpha=1, \quad \text { and } \quad v=0,
\end{aligned}
$$

and the notation:

$$
\Delta=\frac{\partial^{2}}{\partial x^{2}}+\frac{\partial^{2}}{\partial y^{2}}+\frac{\partial^{2}}{\partial z^{2}}, \quad \nabla=\left(\frac{\partial}{\partial x}, \frac{\partial}{\partial y}, \frac{\partial}{\partial z}\right),
$$

where the variables $(x, y, z)$ are the spatial coordinates, $T$ is the temperature, $\alpha$ the concentration of the reaction product, $v$ the mean velocity, $p$ the pressure, $\kappa$ the coefficient of thermal diffusivity, $q$ the adiabatic temperature rise, $\rho$ an average value of density, $v$ the coefficient of kinematic viscosity, $\gamma$ the unit vector in the $\mathrm{z}$-direction (upward), $\beta$ the coefficient of thermal expansion, $g$ the gravitational acceleration, $T_{0}$ a mean value of temperature, $T_{i}$ the initial temperature and $T_{b}=T_{i}+q$ the temperature of the burned mixture. We suppose that the chemical reaction is one-step of zero order. Thus, the reaction rate is of the form:

$$
W=k(T) \phi(\alpha) \quad \phi(\alpha)=\left\{\begin{array}{lll}
1 & \text { if } & \alpha<1, \\
0 & \text { if } & \alpha=1,
\end{array}\right.
$$

in which the temperature dependence of the reaction rate is given by the Arrhenius Law $k(T)=k_{0} \exp \left(-E / R_{0} T\right)$ where $k_{0}$ is the pre-exponential factor, $E$ is the activation energy (assumed to be large) and $R_{0}$ the universal gas constant. The liquid is incompressible and we neglect the term of diffusivity in the concentration equation such that the diffusivity coefficient is very small comparing with the thermic diffusivity coefficient.

We introduce the dimensionless spatial variables:

$$
\begin{gathered}
x_{1}=\frac{x c_{1}}{\kappa}, \quad y_{1}=\frac{y c_{1}}{\kappa}, \quad z_{1}=\frac{z c_{1}}{\kappa}, \\
t_{1}=\frac{t c_{1}^{2}}{\kappa}, \quad p_{1}=\frac{p}{c_{1}^{2} \rho}, \quad c_{1}=\frac{c}{\sqrt{2}}, \\
v_{1}=\frac{v}{c_{1}}, \quad \theta=\frac{T-T_{b}}{q}, \quad \text { and } \quad c^{2}=\frac{2 k_{0} \kappa R_{0} T_{b}^{2}}{q E} \exp \left(-\frac{E}{R_{0} T_{b}}\right)
\end{gathered}
$$

where $c$ denotes the velocity of the propagation of the stationary front, which can be calculated asymptotically for large Zeldovich number. For convenience, we drop the subscribes in the velocity, pressure and the other variables. In this case, the system with the boundary conditions (1)-(6) reads:

$$
\frac{\partial \theta}{\partial t}+(v \cdot \nabla) \theta=\Delta \theta+Z \exp \left(\frac{\theta}{Z^{-1}+\delta \theta}\right) \phi(\alpha)
$$

$$
\begin{gathered}
\frac{\partial \alpha}{\partial t}+(v \cdot \nabla) \alpha=Z \exp \left(\left(\frac{\theta}{Z^{-1}+\delta \theta}\right) \phi(\alpha)\right. \\
\frac{\partial v}{\partial t}+(v . \nabla) v=-\nabla p+P \Delta v+P R\left(1+\lambda_{1} \sin \left(\mu_{1} t\right)+\lambda_{2} \sin \left(\mu_{2} t\right)\right)\left(\theta+\theta_{0}\right) \gamma,
\end{gathered}
$$

$$
\operatorname{div}(v)=0,
$$

with the boundary conditions :

$$
\begin{gathered}
z \rightarrow+\infty, \quad \theta=-1, \quad \alpha=0, \quad \text { and } \quad v=0, \\
z \rightarrow-\infty, \quad \theta=0, \quad \alpha=1, \quad \text { and } \quad v=0 .
\end{gathered}
$$

Here $P=\frac{v}{\kappa}$ is the Prandtl number, $R=g \beta q \kappa^{2} /\left(v c^{3}\right)$ is the Rayleigh number, $Z=q E / R_{0} T_{b}^{2}$ is the Zeldovich number, $\delta=R_{0} T_{b} / E, \theta_{0}=\left(T_{b}-T_{0}\right) / q, \mu_{1}=2 \kappa \sigma_{1} / c^{2}$ and $\mu_{2}=$ $2 \kappa \sigma_{2} / c^{2}$.

\section{The interface problem}

To study the problem analytically we reduce it to a singular perturbation problem where the reaction zone is supposed to be infinitely narrow, and the reaction term is neglected outside of the reaction zone. This is a conventional approach for combustion problems.

To obtain the interface problem, we perform a formal asymptotic analysis with $\epsilon=Z^{-1}$ taken as a small parameter. The linear stability analysis leads to the following interface problem:

$\underline{z>\zeta}$ (liquid monomer):

$$
\begin{gathered}
\frac{\partial \theta}{\partial t}+(v \cdot \nabla) \theta=\Delta \theta, \\
\alpha=0,
\end{gathered}
$$

$\frac{\partial v}{\partial t}+(v . \nabla) v=-\nabla p+P \Delta v+Q\left(1+\lambda_{1} \sin \left(\mu_{1} t\right)+\lambda_{2} \sin \left(\mu_{2} t\right)\right)\left(\theta+\theta_{0}\right) \gamma$

$$
\operatorname{div}(v)=0,
$$

$z<\zeta$ (solid polymer):

$$
\begin{gathered}
\frac{\partial \theta}{\partial t}=\Delta \theta, \\
\alpha=1, \\
v=0 .
\end{gathered}
$$

$z=\zeta$ (interface):

$$
\left.\theta\right|_{\zeta-0}=\left.\theta\right|_{\zeta+0},
$$

$$
\left.\frac{\partial \theta}{\partial z}\right|_{\zeta=-0}-\left.\frac{\partial \theta}{\partial z}\right|_{\zeta=+0}=\left(1+\left(\frac{\partial \zeta}{\partial x}\right)^{2}+\left(\frac{\partial \zeta}{\partial y}\right)^{2}\right)^{-1} \times \frac{\partial \zeta}{\partial t}
$$




$$
\left.\left(\frac{\partial \theta}{\partial z}\right)^{2}\right|_{\zeta-0}-\left.\left(\frac{\partial \theta}{\partial z}\right)^{2}\right|_{\zeta_{1}+0}=-2 Z\left(1+\left(\frac{\partial \zeta}{\partial x}\right)^{2}+\left(\frac{\partial \zeta}{\partial y}\right)^{2}\right)^{-1} \exp \left(\left.Z \theta\right|_{\zeta}\right)
$$

$$
v_{x}=v_{y}=v_{z},
$$

with the conditions at infinity:

$$
\begin{array}{cc}
z=-\infty: & \theta=0, \quad v=0 \\
z=+\infty: & \theta=-1, \quad v=0 .
\end{array}
$$

\section{Numerical results}

We seek the solution of the interface problem (13)-(25) in the form:

$$
\theta=\theta_{s}+\tilde{\theta}, \quad p=p_{s}+\tilde{p}, \quad v=v_{s}+\tilde{v}
$$

where $\tilde{\theta}, \tilde{p}$ and $\tilde{v}$ are, respectively, small perturbation of temperature, pressure and velocity.

We consider the perturbation in the form:

$$
\begin{gathered}
\hat{\theta}_{i}=\theta_{i}\left(z_{2}, t\right) \exp \left(j\left(k_{1} x+k_{2} y\right),\right. \\
\hat{v}_{z i}=v_{z i}\left(z_{2}, t\right) \exp \left(j\left(k_{1} x+k_{2} y\right),\right. \\
\xi=\epsilon_{1}(t) \exp \left(j\left(k_{1} x+k_{2} y\right),\right.
\end{gathered}
$$

where $k_{i}, i=1,2$ are, respectively, the wave numbers (in $x$ and $y$ directions).

Finally, the propagating reaction front problem will be reduced to the following system:

$$
\left\{\begin{aligned}
\frac{\partial w}{\partial t}= & P\left(w^{\prime \prime}-k^{2} w\right)+u w^{\prime}+P R k^{2} \theta\left(1+\lambda_{1} \sin \left(\mu_{1} t\right)\right. \\
& \left.+\lambda_{2} \sin \left(\mu_{2} t\right)\right) \\
-w= & v^{\prime \prime}-k^{2} v \\
\frac{\partial \theta}{\partial t}= & \theta^{\prime \prime}+u \theta^{\prime}-k^{2} \theta+u \exp \left(-u z_{2}\right) v
\end{aligned}\right.
$$

with

$$
z=0, \quad \theta^{\prime}=-u \theta ; \quad v=v^{\prime}=0
$$

and

$$
z=L, \quad \theta=v=w=0
$$

Figure 1 shows the critical Rayleigh number as a function of the amplitude $\lambda_{2}$ of vibrations for $P=10, k=1.5$, $\lambda_{1}=5, \mu_{1}=5$ and for different frequencies ratio. It can be observed that as the frequencies ratio increases, the stability of reaction front is gained, especially in certain interval corresponding to small values of the amplitude $\lambda_{2}$. The stability region is reduced for large values of the vibration amplitude $\lambda_{2}$.

In Figure 2 the critical Rayleigh number versus the frequency $\mu_{1}$ is shown for the given values $P=10, k=1.5$, $\lambda_{1}=\lambda_{2}=5$ and for different frequencies ratio. It can be seen from this figure that in the absence of modulation the classical result of the unmodulated case is found [2]. It is interesting to notice that the classical critical value $R_{C} \simeq 83$ is also obtained for values of the frequency $\mu_{1}$

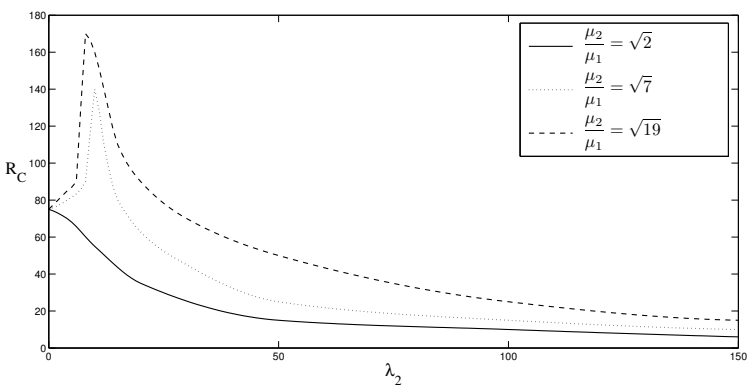

Fig. 1. The critical Rayleigh number versus the amplitude of vibration $\lambda_{2}$ for different frequencies ratio.

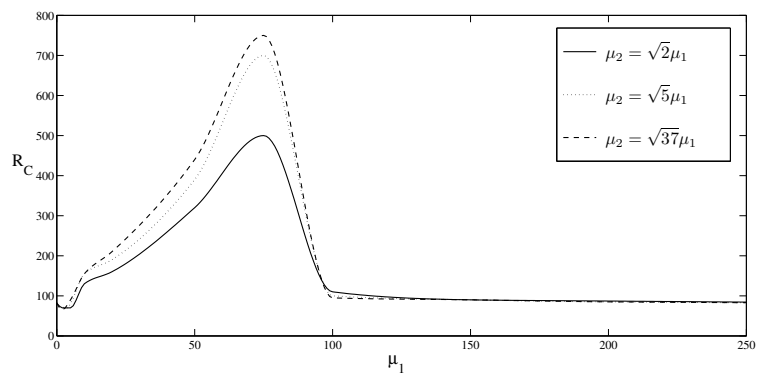

Fig. 2. The critical Rayleigh number versus the frequency of vibration $\mu_{1}$ for different frequencies ratio.

larger than 100. Instead, in the interval of $\mu_{1}$ between 0 and 100 the plots depict that increasing the frequencies ration $\frac{\mu_{2}}{\mu_{1}}$ increases the stability domain of the reaction front. Beyond $\mu_{1} \simeq 100$ the frequency ratio has no effect on the convective instability.

\section{Conclusion}

In this work, we have studied the influence of the QP vibration on the convective instability of polymerization front with liquid reactant and solid product. For this purpose, we have considered the model which includes the heat equation, the concentration equation and the NavierStokes ones under boussinesq approximation.

The Zeldovich Frank-Kamenetskii method, called also narrow zone approximation has been used assuming the reaction occurs in a small zone when the activation energy is high. We have performed an asymptotic analysis to determine the interface problem and the solution has been chosen as a perturbed stationary solution. The results based on numerical integration of the reduced reaction front system of equations show the strong dependence of the convective instability threshold on the amplitudes and the incommensurate frequencies ratio.

\section{References}

1. P.M. Goldfeder, V.A. Volpert. V.M. Ilyashenko, A.M. Khan, J.A. Pojman, S.E. Solovyov. Mathematical modeling of free-radical polymerization fronts. The Journal of Physical Chemistry B, 101, 3474-3482 (1997). 
2. K. Allali, J. Pojman, V. Volpert. Influence of vibrations on convective instability of polymerization fronts. J. of Engineering Mathematics, 41, 13-31 (2001).

3. K. Allali, M. Belhaq, K. El Karouni. Influence of quasi-periodic gravitational modulation on convective instability of reaction fronts in porous media. Commun. Nonlinear Sci. Numer. Simul. 17, 1588-1596 (2012).

4. T. Boulal, S. Aniss, M. Belhaq, R.H. Rand. Effect of quasiperiodic gravitational modulation on the stability of a heated fluid layer. Phys. Rev. E, 52(76):56320 (2007).

5. T. Boulal, S. Aniss, M. Belhaq, A. Azouani. Effect of quasi-periodic gravitational modulation on the convective instability in Hele-Shaw cell. Int. J. Non Linear Mech. 43, 852-857 (2008).

6. T. Boulal, S. Aniss, M. Belhaq. Quasiperiodic gravitational modulation of convection in magnetic fluid, Thermal non-equilibrium. In: Wiegand S, Köhler W, Dhont JKG. editors. Lecture notes of the 8th international meeting of thermodiffusion, 9-13 June, 2008, Bonn, Germany; 2008, (300) p. ISBN: 978-3-89336523-4.

7. R.H. Rand, K. Guennoun, M. Belhaq. 2:2:1 Resonance in the quasi-periodic Mathieu equation. Nonlinear Dynam. 31, 367-374 (2003).

8. S.M. Sah, G. Recktenwald, R.H. Rand, M. Belhaq. Autoparametric quasiperiodic excitation. Int. J. Nonlinear Mech. 43, 320-327 (2008).

9. V1. Volpert, Vi. Volpert, V. Ilyashenko, J. Pojman. Frontal polymerization in a porous medium. Chem. Eng. Sci. 53, 1655-1665 (1998).

10. M. Abdul Mujeebu, M.Z. Abdullah, M.Z. Abu Bakar, A.A. Mohamad, R.MN. Muhad, M.K. Abdullah. Combustion in porous media and its applications. A comprehensive survey. J. Environ. Manage. 90:2287312 (2009). 